\title{
Teacher: But Not Student Rating of the Pedagogic and Social Climate in School Predicts Adolescents' Academic Aspirations
}

\author{
Melody Almroth $^{1}$ (D) Krisztina D. László ${ }^{1} \cdot$ Kyriaki Kosidou $^{1,2} \cdot$ Maria Rosaria Galanti $^{1,2}$
}

Accepted: 5 January 2021 / Published online: 27 January 2021

(c) The Author(s) 2021

\begin{abstract}
Background High academic aspirations relate to higher achievement and better mental health, but less is known about how these aspirations are formed in relation to the educational context.

Objective This study aims to investigate the relationship between overall school climate, with particular concern for the dimensions of school level expectations and support as rated by both teachers and students and adolescent academic aspirations.

Methods Multilevel logistic models for repeated measures were used in order to investigate the relationship between measures of school climate and adolescents' academic aspirations. Three annual waves of questionnaire data were used to obtain aggregated teacherand student-rating of school climate, including specific dimensions of teacher expectations and support.

Results Positive teacher-rated overall school climate was associated with an increased odds of adolescents aiming at a university education rather than at a lower one (adjusted OR 1.36, 95\% CI 1.14-1.63 for the intermediate tertile; OR 1.39, 95\% CI 1.14-1.70 for the highest tertile). A similar trend was found for the teacher-rated measures of expectations and student focus, but not for any of the student-rated school climate measures.

Conclusion A positive school climate rated by teachers appears to predict adolescents' university aspirations. Future research should clarify which aspects of the school climate may influence adolescent academic aspirations from the students' perspective.
\end{abstract}

Keywords Adolescence $\cdot$ School climate $\cdot$ Academic expectations $\cdot$ Academic aspirations

Supplementary Information The online version contains supplementary material available at (https:// doi.org/10.1007/s10566-021-09601-9).

Melody Almroth

melody.almroth@ki.se

1 Karolinska Instututet Department of Global Public Health, Solnavägen 1E, Floor 6, 11365 Stockholm, Sweden

2 Center for Epidemiology and Community Medicine, Stockholm County Council, Solnavägen 1E, Floor 6, 11365 Stockholm, Sweden 


\section{Introduction}

High academic aspirations refer to a person aiming to stay in education longer and correlate with more positive mental health in terms of both internalizing (Almroth et al. 2018) and externalizing problems (Almroth et al. 2019) as well as high academic achievement (Khattab 2015). However, a better understanding of factors that impact the development of adolescent's academic aspirations is needed before this knowledge can be translated into strategies aimed at the improvement of academic trajectories while preserving mental well-being.

The majority of previous studies on adolescent academic expectations or aspirations focused on the influence of socio-demographic characteristics such as gender (Kirk et al. 2012; Mello 2008), race or ethnicity (Irvinet al. 2016; Nitardy et al. 2015), or socioeconomic status (Chykina et al. 2016), that is, characteristics that are not modifiable. Less attention has been given to potentially modifiable contextual factors such as those related to the educational environment.

According to Ecological Systems Theory, a developing person lies at the center of several nested and inter-related systems ranging from the more proximal microsystems that an individual interacts with directly to the more distal societal and cultural norms (Bronfenbrenner and Morris 2006). School is an important microsystem and a major context for cognitive development, health promotion and prevention of risk factors affecting adolescents' health. Furthermore, school is assumed to reduce inequality in developmental opportunities in relation to socio-demographic characteristics. For example, a systematic review of 78 studies found that a positive school climate consistently minimized the gap in academic achievement between high and low socioeconomic status students (Berkowitz et al. 2016).

Measurements of school climate vary extensively between studies, but they generally encompass issues like safety, relationships between students and staff, teaching approaches, and the physical school facilities (Hultin et al. 2018; Thapa et al. 2013). Positive school climates in these domains are associated with favorable academic outcomes, less truancy, and improved student self-concept (Haynes et al. 1997). Less is known, however, about how the school climate specifically influences students' academic aspirations.

Several previous studies found an association between various aspects of a positive school climate and high academic aspirations (Israelashvili 1997; Madarasova-Geckova et al. 2010; Marjoribanks 2002). However, another study found students' perception of school climate, defined by feelings of safety and belonging at school, to be negatively associated with academic aspirations, and attributed this unexpected finding to an antieducation ethos in the English context (Frostick et al. 2016). Other studies have considered school climate in relation to constructs which may be similar or closely related to academic aspirations. For instance, some studies have found a positive relationship between aspects of school climate and student engagement in school (Bear et al. 2018; Wang and Holcombe 2010; Yang et al. 2018) or future goals (Lindstrom-Johnson et al. 2016).

All of the above-mentioned studies relied only on student perception of school climate, and the majority did not measure school climate on an aggregate level. Because school climate is based on a collective experience, it may be better understood through multiple and aggregate perspectives rather than individual ratings (Grosin 2004). It is important to understand the school environment as a context rather than an individual 
experience. Measuring school climate and academic aspirations using the same informant may also result in cognitive bias, where individuals with a tendency towards lower aspirations may evaluate their school climates more negatively rather than the other way around.

One particular dimension of school climate which may be important for academic aspirations is the academic and behavioral expectations conveyed at the overall school level by the teaching body and by the school leadership; an aspect that remains to be elucidated. In fact, the existing empirical evidence only concern individual teachers' expectations as predictors of their own students' academic achievement (Benner and Mistry 2007; Gregory and Huang 2013) and perception of abilities (Rubie-Davies 2006). One study has also found that teachers' expectations predicted their own students' academic aspirations (Irvin et al. 2016). However, expectations as a whole-school vocation are a different dimension compared to expectations conveyed in a classroom environment by individual teachers. It is important to further understand how expectations are conveyed on a school level through the school's educational climate and how this relates to the formation of academic aspirations.

Despite their heterogeneity, most measures of school climate include at least one aspect of teacher support or quality of the student-teacher relationships (Berkowitz et al. 2016). Some studies have found that perceived support from teachers and counselors in terms of availability and approachability were related to higher student academic expectations or aspirations (Melkman et al. 2016; Smith et al. 2016), indicating that teacher support may be a dimension of school climate of paramount importance to the development of students' academic aspirations.

Sweden provides a unique context for investigating educational environments and the formation of academic aspirations because the school system was rapidly decentralized during the 1990s (Wikström 2006). At the same time, a policy change allowed the emergence of privately run but publicly funded independent schools. This has resulted in greater inequality between schools in terms of students' socioeconomic background (Berhanu 2010; Wikström 2006) and academic achievements (OECD 2015). Thus, it is important to consider whether the relationship between school climate factors and academic aspirations may differ according to some of these structural and functional aspects of Swedish schools, such as public or private management and compositional sociodemographic characteristics. Specifically, whether school climate may be more important to the development of academic aspirations in certain sociodemographic contexts. Furthermore, the end of 9th grade represents an educational transition where students decide if they want to continue their education after compulsory school, and if so, if they would like to follow an academic or vocational track (OECD 2015). Thus, the final years of compulsory school are an important time for the development of academic aspirations. Additionally, Sweden has fewer financial barriers to higher education compared to other contexts, perhaps making student's academic aspirations more malleable and less dependent on their family's financial circumstances.

The present study aims to investigate the extent to which a positive school climate, measured as an aggregated and contextual factor is related to adolescents' university aspirations and engagement with future goals. The study uses multiple informants, in that both the teacher and student collective experiences of school climate are considered, as well as its specific dimensions of school-level expectations and support. Further, this study aims to explore whether specific structural and functional characteristics of schools moderate the relationships between school climate and adolescents' academic aspirations. 
Because proximal environments, and more specifically, school climates, have been found to be related to academic outcomes, we predict that more positive school climates with higher expectations and greater student support will relate to university aspirations and higher student engagement. Furthermore, we hypothesize that both teacher and student higher ratings of school climate will predict higher academic aspirations, but these perspectives may represent important differences which are neglected in current modern school climate research which only captures the students' perspective. Finally, because of specific structural components of the Swedish school system, we hypothesize that school climate may show stronger associations in schools which may be more disadvantaged in Sweden's current academic system in terms of school management and demographic composure.

\section{Method}

\section{Study Population and Design}

This study builds on the KUPOL longitudinal study focusing on the relationship between school factors and adolescent mental health in Sweden. Extensive information regarding the study design and the data collection procedure of the KUPOL study has previously been published (Galanti et al. 2016) and will be briefly summarized here. The parents of 3959 7th grade adolescents (age 13) from 101 Swedish primary schools gave informed consent for their children's participation in the study during the 2013/2014 or 2014/2015 school years. Parents and children answered questionnaires at baseline and during two subsequent annual follow-ups. Data assessing school climate were collected in the same schools during each schoolyear using anonymous questionnaires given to all teachers and to all 9th grade students. Therefore, the student informants on school climate did not encompass the cohort participants apart from the last year. Ninth grade students were chosen because they had attended the schools the longest and were thought to be able to give the most informed rating of the school climate. The school climate assessment was answered by 4542 teachers and 11,282 students during the years corresponding to the first wave of data collection.

The KUPOL study was approved by the Stockholm Ethics Review Board (reference numbers: 2012/1904-31/1 and 2016/1280-32). The authors declare that they have no conflict of interest.

\section{Measures}

School climate was assessed using the PESOC (Pedagogical and Social Climate) instrument. This instrument, rooted in school effectiveness research, was developed in Sweden by Lennart Grosin (2004) with the intention of measuring aspects of school cultural, structural, and social climate based on the schools ethos, norms, and interactions between staff, students, and families (Hultin et al. 2018). The PESOC instrument has two versions: one capturing the teachers' perspective, and one assessing the students' perspective. Both versions have previously shown high validity and reliability (Dimitrova et al 2016; Hultin et al. 2016, 2018).

More information about the scales and the items they contain have been described in detail elsewhere (Hultin et al. 2016, 2018). 
In summary, the teacher version contains 67 items divided into 11 subscales: expectations; teacher agreement about goals, norms, and rules; student focus; assumption about student's ability to learn; communication between school and home; teachers' interaction and cooperation; teachers confidence and professional development; teaching activities; evaluation of academic progress of the student; principals pedagogic leadership; and involvement and support of school management. The student version includes 53 items in eight subscales: expectations; teacher norms; teacher support; teaching activities; student participation; school environment; school and home; and school leadership. All items for both versions of the instrument were answered on a four-point scale ranging from strongly disagree to strongly agree. There was an additional option for I don't know which was coded as missing.

For this study we used scores derived from both instruments as follows. From the teacher's instrument we derived the total PESOC score using all available items. The Cronbach's alpha for the total teacher-rated PESOC was 0.96. We also used scores from two specific subscales: expectations and student focus. The expectations subscale contains four items regarding academic and behavioral expectations. A sample item is "Our principal has high demands and expectations for pupil academic results." The student focus subscale contains six items and evaluates student and teacher relationships, with a sample item being, "Social relationships between teachers and pupils are good at this school". The Cronbach's alphas for the teacher-rated expectations subscale and the student focus subscale were 0.64 and 0.68 respectively.

From the student instrument, we also derived a total school climate score using all 53 available items. The Cronbach's alpha for the total student-rated PESOC was 0.96. We also derived the score from the student-rated expectations subscale containing four items and from the teacher support subscale containing five items. The Cronbach's alphas for these subscales were 0.59 and 0.77 , respectively.

All scores from the PESOC instruments were calculated as an average score of the corresponding items, where a higher score is equivalent to a more positive rating. Because the teacher-rated student focus subscale, the student-rated total school climate score, and the student-rated academic and disciplinary expectations scores had a range smaller than one, we categorized all PESOC scales and subscales according to their tertile distributions.

Students' academic aspirations were assessed annually using two measures. Students were asked how far they wanted to go in school with the original response alternatives "I don't know", "high school vocational track", "high school theoretical track", and "university". This variable was dichotomized to reflect whether or not students wanted to attend university. The three non-university categories have shown consistent similarities to each other compared to the university category in previous analyses (Almroth et al. 2018, 2019).

Students' aspirations and future goals were also assessed using the Future aspirations and Goals (FG) subscale of the validated Student Engagement Instrument (Appleton et al. 2006; Betts et al. 2002). This subscale rests on five items regarding plans for future education, the importance of education, and hopefulness about the future. Items were answered on a five-point scale ranging from strongly disagree to strongly agree. Following the protocol for the scale (Check and Connect Study 2015), the scale score is calculated as an average of the five items (ranging from one to five), where a higher score indicates higher aspirations. Because this variable did not meet the assumptions of normal distribution, a dichotomous variable was derived using the median of 4.6 as the cutoff point.

Other school related variables were obtained from the SIRIS online database of the Swedish National Agency for Education (https://www.skolverket.se/skolutveckling/stati stik). These included school ownership (public versus privately run school); percent of 
students with parents with a university education; and percent of students born outside of Sweden.

Other individual-level variables were also measured. Parents' education was assessed from the parents' questionnaire and was categorized as "at least one parent attended university" versus "neither parent attended university". Parents' country of birth, assessed the same way, was categorized as "both parents born in Sweden" versus "at least one parent born outside of Sweden". Adolescents' psychiatric diagnoses were obtained through linkage to the child and youth outpatient register (BUP). This was then coded as "any psychiatric diagnosis before baseline data collection" versus "no psychiatric diagnosis before baseline data collection".

\section{Statistical Analysis}

Differences in the distribution of baseline characteristics according to adolescent's baseline aspirations and FG scale were assessed using chi-square tests for categorical variables, and Kruskal-Wallis tests for continuous variables.

In order to investigate whether higher rated school climate factors predicted higher academic aspirations, and whether this differed depending on the student or teacher ratings, separate multilevel logistic regression models with repeated measures were built to measure the relationship between each of the PESOC scales and subscales and university aspirations or the FG scale. Observations were used from all three yearly assessments for both individual and school-level measures, clustered within individuals (level 1) and schools (level 2). Individuals contributed to one or more time point given that they had complete information on the exposure and outcome for at least one of the data collection waves. Models were built using the GLIMMIX procedure using SAS Enterprise Guide 7.1. Models were first built containing no predictors for each of the two outcomes (university aspirations and FG scale) in order to calculate the intra-class correlations. Unadjusted models were then built for each of the school climate predictors separately according to the two outcomes (Model 1). All models were then adjusted for parental education and birth country at the individual level (Model 2). These potential confounders were chosen because they are likely to influence both the choice of school and the adolescent's aspirations, but do not lie on the causal pathway between the two.

To investigate whether the relationships between school climate factors and adolescent aspirations differed according to structural and functional factors at the school level, that is, whether school climate factors were more important under specific circumstances, models were stratified according to school ownership, proportion of students with parents with a university education, and proportion of students born outside of Sweden categorized by the corresponding tertile distribution. Formal tests of interaction were also performed using interaction terms.

Additionally, stratified models were built according to the individual sociodemographic factors of parents' education, and parents' country of birth. The purpose of this being to explore the extent to which relationships differed according to each factor rather than only adjusting for these factors in the main models.

Sensitivity analyses were also performed to assess the robustness of the results. In order to avoid the risk of reverse associations (i.e. that students' selection of school is dependent on their mental health which may be related to their academic aspirations, thus predicting the climate of their school), we conducted all analyses after exclusion of students with a 
psychiatric diagnosis prior to entry into the cohort. In order to investigate the impact of school response rate, we repeated the analyses after exclusion of schools with less than $30 \%$ response for the teacher or student PESOC ratings.

The percent of missing data in each covariate ranged from 2 to $15 \%$ during all three annual waves of data collection. Using multilevel models for repeated measures was a deliberate strategy for making the best use of the available data.

\section{Results}

Table 1 reports school and individual level predictors of academic aspirations and FG scale score. Both or at least one of these dimensions of aspirations were higher among students who attended private schools, schools with a higher average proportion of highly educated parents, and schools with a higher average proportion of students born outside of Sweden. Individual predictors of high academic aspirations and FG score were having at least one parent with university education or having at least one parent born outside of Sweden. No differences were apparent between adolescents with or without prior psychiatric diagnoses.

The intra-class correlation at the school level was $10 \%$ for university aspirations and $6 \%$ for the FG score, indicating that $10 \%$ and $6 \%$ of the variability for university aspirations and FG score is explained at the school level. Table 2 displays the associations between school climate scores and student university aspirations while the corresponding associations with the FG score are shown in Table 3. Attending a school where the teacherbased school climate score was in the two higher tertiles compared to the lowest tertile was associated with an increased odds of students endorsing aspirations of university education (adjusted OR 1.36 95\% CI 1.14-1.63 for the intermediate tertile; OR 1.39 95\% CI 1.14-1.70 for the highest tertile). The teacher-rated expectations and student focus subscales showed similar associations. Student-rated school climate was not associated with individual academic aspirations, nor were the expectations and teacher support subscales (Table 2). No significant associations were found between any of the school climate scales and the FG scale score (Table 3).

Stratified analyses and models including interaction terms did not generally reveal any moderating effects according to school ownership, percent of highly educated parents, or percent of students born outside of Sweden. Two of the student-rated measures of school climate and university aspirations seemed to vary according to the percent of students with parents born outside of Sweden. Even in this case, however, there were no clear and interpretable patterns (Tables S3-S5). Similarly, there were no clear moderating effects according to parental birth country and parental education at the individual level (not shown).

All associations were similar when excluding adolescents with a psychiatric diagnosis prior to baseline; or adolescents attending a school with a response rate to the PESOC climate rating instrument below 30\% (Tables S1 and S2).

\section{Discussion}

In this large, multi-informant, longitudinal study of school-level predictors of Swedish adolescents' aspirations, we found that higher teacher-rated school climate, and the specific dimensions of expectations and student focus were associated with adolescents' self-reported university aspirations. Surprisingly, no associations were found between the 


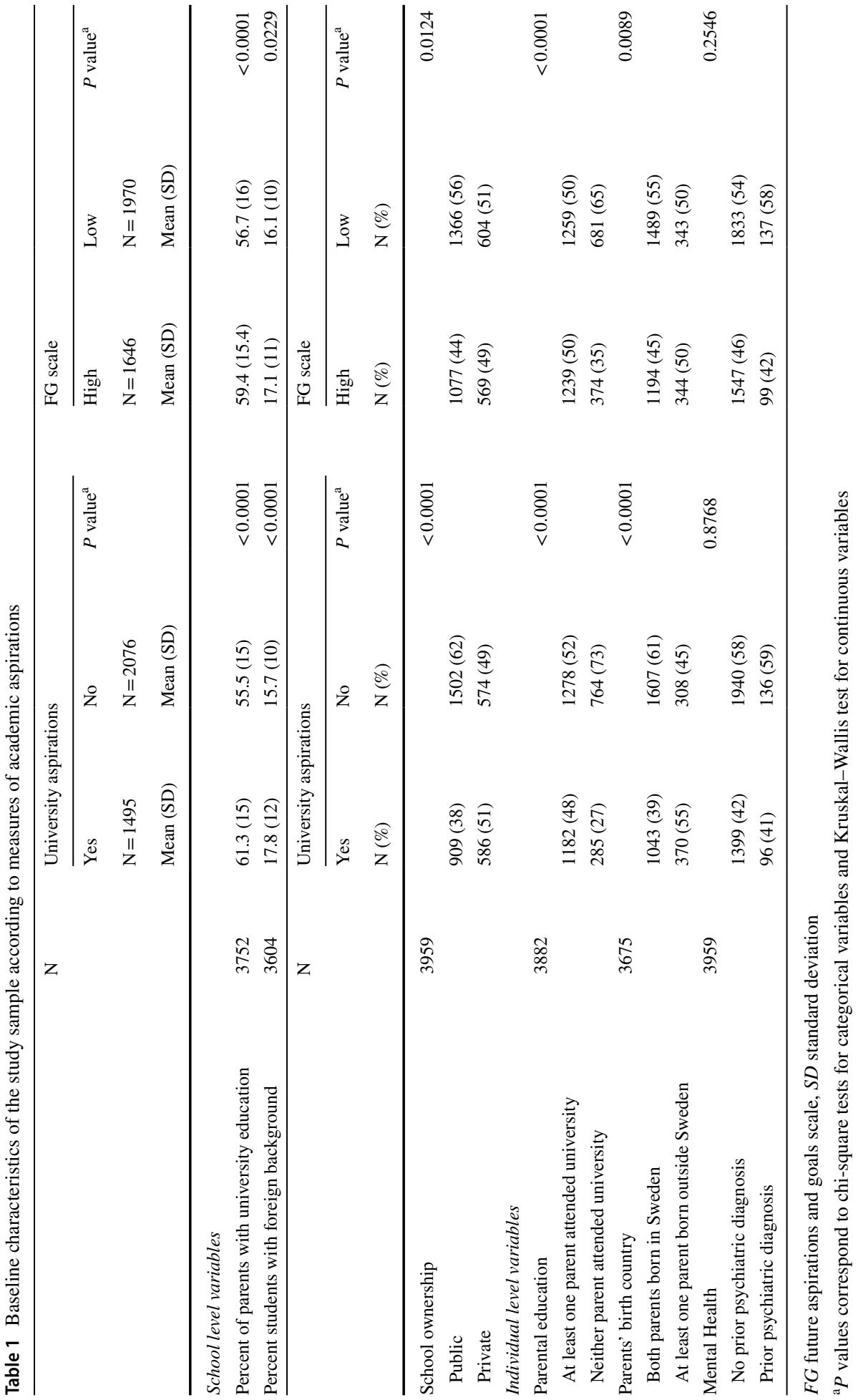


Table 2 Odds ratio and $95 \%$ confidence intervals for adolescent university aspirations according to school climate measures

\begin{tabular}{|c|c|c|}
\hline \multirow[t]{2}{*}{ Exposure } & \multicolumn{2}{|l|}{ OR $(95 \% \mathrm{CI})$} \\
\hline & Model $1^{\mathrm{a}}$ & Model $2^{\mathrm{b}}$ \\
\hline \multicolumn{3}{|c|}{ Teacher-rated total PESOC } \\
\hline Low & 1 & 1 \\
\hline Medium & $1.42(1.19-1.70)$ & $1.36(1.14-1.63)$ \\
\hline High & $1.42(1.16-1.73)$ & $1.39(1.14-1.70)$ \\
\hline \multicolumn{3}{|c|}{ Teacher-rated expectations } \\
\hline Low & 1 & 1 \\
\hline Medium & $1.15(0.97-1.36)$ & $1.17(0.99-1.38)$ \\
\hline High & $1.34(1.11-1.61)$ & $1.37(1.13-1.66)$ \\
\hline \multicolumn{3}{|c|}{ Teacher-rated student focus } \\
\hline Low & 1 & 1 \\
\hline Medium & $1.20(1.02-1.40)$ & $1.18(1.00-1.38)$ \\
\hline High & $1.38(1.12-1.71)$ & $1.36(1.10-1.68)$ \\
\hline \multicolumn{3}{|c|}{ Student-rated total PESOC } \\
\hline Low & 1 & 1 \\
\hline Medium & $1.07(0.94-1.22)$ & $1.06(0.92-1.22)$ \\
\hline High & $1.07(0.90-1.28)$ & $1.07(0.89-1.28)$ \\
\hline \multicolumn{3}{|c|}{ Student-rated expectations } \\
\hline Low & 1 & 1 \\
\hline Medium & $1.02(0.88-1.18)$ & $1.01(0.87-1.17)$ \\
\hline High & $1.13(0.96-1.34)$ & $1.12(0.95-1.33)$ \\
\hline \multicolumn{3}{|c|}{ Student-rated teacher support } \\
\hline Low & 1 & 1 \\
\hline Medium & $1.11(0.97-1.28)$ & $1.10(0.95-1.27)$ \\
\hline High & $1.09(0.92-1.29)$ & $1.08(0.91-1.27)$ \\
\hline
\end{tabular}

$O R$ odds ratio, $C I$ confidence interval, PESOC pedagogic and social climate instrument

${ }^{a}$ Model 1 is unadjusted

${ }^{\mathrm{b}}$ Model 2 is adjusted for parental education and parental country of birth

student-rated school climate measures and adolescent university aspirations, and none of the school climate measures appeared to predict future aspirations and goals.

Previous studies have found positive school climate in general (Israelashvili 1997; Lindstrom-Johnson et al. 2016; Madarasova-Geckova et al. 2010; Marjoribanks 2002) to be related to academic expectations or aspirations. Several others have pointed to the specific influence of teacher or school support (Melkman et al. 2016; Smith et al. 2016). Others have found a similar relationship between positive school climate and higher student academic engagement (Bear et al. 2018; Wang and Holcombe 2010; Yang et al. 2018). Our study, however, is novel in that we did not use the same student informant to measure both school climate factors and academic aspirations.

One previous study found that student's perceptions of a positive school climate were associated with lower adolescent aspirations, however this particular study focused only on schools in areas of high deprivation and focused on aspects of school climate related to the social rather than academic environment (Frostick et al. 2016). The authors also described 
Table 3 Odds ratio and $95 \%$ confidence intervals for high future aspirations and goals score according to school climate measures

\begin{tabular}{|c|c|c|}
\hline \multirow[t]{2}{*}{ Exposure } & \multicolumn{2}{|l|}{ OR $(95 \% \mathrm{CI})$} \\
\hline & Model $1^{\mathrm{a}}$ & Model $2^{b}$ \\
\hline \multicolumn{3}{|c|}{ Teacher-rated total PESOC } \\
\hline Low & 1 & 1 \\
\hline Medium & $1.09(0.92-1.27)$ & $1.04(0.88-1.23)$ \\
\hline High & $1.03(0.86-1.23)$ & $0.98(0.82-1.17)$ \\
\hline \multicolumn{3}{|c|}{ Teacher-rated expectations } \\
\hline Low & 1 & 1 \\
\hline Medium & $1.06(0.91-1.23)$ & $1.01(0.87-1.18)$ \\
\hline High & $1.01(0.85-1.20)$ & $0.97(0.82-1.15)$ \\
\hline \multicolumn{3}{|c|}{ Teacher-rated student focus } \\
\hline Low & 1 & 1 \\
\hline Medium & $0.98(0.84-1.13)$ & $0.95(0.82-1.10)$ \\
\hline High & $1.08(0.89-1.29)$ & $1.01(0.84-1.22)$ \\
\hline \multicolumn{3}{|c|}{ Student-rated total PESOC } \\
\hline Low & 1 & 1 \\
\hline Medium & $0.97(0.85-1.10)$ & $0.99(0.87-1.12)$ \\
\hline High & $1.09(0.93-1.28)$ & $1.11(0.95-1.31)$ \\
\hline \multicolumn{3}{|c|}{ Student-rated expectations } \\
\hline Low & 1 & 1 \\
\hline Medium & $0.94(0.82-1.08)$ & $0.95(0.82-1.09)$ \\
\hline High & $1.05(0.90-1.22)$ & $1.06(0.91-1.24)$ \\
\hline \multicolumn{3}{|c|}{ Student-rated teacher support } \\
\hline Low & 1 & 1 \\
\hline Medium & $1.02(0.90-1.17)$ & $1.05(0.92-1.20)$ \\
\hline High & $1.02(0.88-1.19)$ & $1.04(0.89-1.21)$ \\
\hline
\end{tabular}

$O R$ odds ratio, $C I$ confidence interval, PESOC pedagogic and social climate instrument

${ }^{a}$ Model 1 is unadjusted

${ }^{\mathrm{b}}$ Model 2 is adjusted for parental education and parental country of birth

an anti-education ethos in this context, where students may be generally less likely to report high aspirations (Frostick et al. 2016). Perception of school environment and aspirations were, again, reported by the same adolescents, and school level differences were not considered.

Our findings that teacher-rated positive overall school climate and the specific dimensions of teachers' expectations and student focus are related to adolescents' aspirations of university education are in line with Ecological Systems Theory, which describes the complex interaction between a person and his or her environment (Bronfenbrenner 1979). Particularly, microsystems such as school shape psychological and behavioral development of individuals through rules and norms (Bronfenbrenner 1979). Stage-Environment Fit Theory also specifies that suboptimal learning environments fail to meet the needs of adolescents appropriate for their development, which results in a mismatch between the person and environment and leads to declining motivation (Roeser 2005). Thus, schools with higher rated total school climate, expectations, and student focus may be those with higher attention for students' developmental 
stages, in the end turning into higher aspirations. A small body of research has focused specifically on the authoritative school climate which is defined by both high expectations and high levels of teacher support (Konold and Cornell 2015). These aspects have been found to be related to better academic outcomes (Cornell et al. 2016; Jia et al. 2016), thus offering theoretical support to our findings that these particular aspects are important to the development of academic aspirations in our sample.

Our finding that student-rated school climate did not appear to be related to adolescent academic aspirations was somewhat surprising. One explanation might be that teacher reports capture some of the behind the scenes processes that may not be clear to students, such as interactions between staff, and school management, thus having a better assessment of how the school functions as a whole. Students may also be more likely to rate their school's climate based on their individual experiences of specific teachers and their classroom climate, while teachers may more accurately assess the overall school climate, therefore making the students' aggregated measure more heterogeneous and more difficult to interpret. Additionally, teachers may derive their judgement through their experience with multiple schools, while most students do not have this perspective. These possible interpretations are particularly relevant considering that the vast majority of modern school climate research focuses exclusively on the student perspective (Berkowitz et al. 2016). Our results point to the importance of the teachers' perspective of school climate in relation to academic aspirations, an aspect which has often previously been neglected.

Though several studies found school climate to be related to academic engagement (Wang and Holcombe 2010; Yang et al. 2018), we saw consistently null associations when we used the scale which measures future goals and cognitive engagement with school. This instrument may represent more intrinsically motivated aspirations compared to the more concrete and externally driven goal of university attendance. Because these previous studies relied on the same informant for both school climate measures and engagement in school, this association may be explained by correlation of individual characteristics or by reverse causality (i.e., intrinsic motivation determines positive school rating). It is possible that the school environment may more easily influence specific extrinsic goals such as attending university compared to intrinsic motivation related to student engagement. It is an interesting finding in itself that the majority of students in this sample reported being highly engaged in school, with the median score being quite close to the highest possible score. This may indicate that students' internal engagement is influenced by other factors outside of the climate of the schools they attend.

Tendencies in the associations between the measures of school climate and adolescent academic aspirations were rather consistent even after stratifying for a variety of individual and school level socio-demographic characteristics, as well as in sensitivity analyses based on the mental health of the participants prior to baseline and on the proportion of responders. That the overall relationship between school climate and academic aspirations may be equally important regardless of school management and demographic composition is of particular interest in the Swedish context given that policy changes have affected school management and differences in school demographic makeup (Wiborg 2015; Wikström 2006). 


\section{Strengths and Limitations}

This study used a large sample of Swedish adolescents, multiple informants, and collected data over three yearly follow ups. Additionally, school climate was analyzed on an aggregated level involving both teacher and student perspectives. There were also several limitations. The recruitment of participating schools and students resulted in a selected sample, with an over-representation of high socio-economic status schools and families, which may have restricted the range of exposures and further limited the power to detect modest effects (Galanti et al. 2016). For example, the majority of students reported having quite high future aspirations and goals. Also, the generalizability of the findings may be limited by the selection of this sample in that there may be some variation in results when less educated and immigrant parents are better represented. However, stratified analyses according to parents' education and birth country at the individual level did not reveal obvious differences in associations in our sample. Sweden also has unique educational policies which may not be directly comparable with other national contexts. Additionally, although we adjusted the associations for several potential confounders, we cannot rule out residual confounding due to unmeasured factors influencing both individuals' choice of school and their academic aspirations.

\section{Conclusion}

A positive school climate rated by teachers appears to be important for adolescents' wanting to achieve university education. This is important considering that the teachers' perspective is often neglected in related research. Future research should focus on refining dimensions of the school climate from the students' perspective that may influence youth academic aspirations. Also, interventions targeting the school climate are needed to determine whether this can actually be improved and whether this improvement reflects an increase of academic aspirations among young people as well as subsequent trajectories of academic achievements and mental health. Because of its potentially important implications for the development of adolescents academic aspirations, school climate should be an important part of the discussion in terms of school planning and evaluation. Lastly, it may be important to find ways to better understand and support students who do not have university aspirations.

Acknowledgements We would like to thank the students, parents, and schools who participated in the KUPOL study. We would also like to thank the administrative staff for the KUPOL project who have made our research possible.

Author contributions MRG is the principal investigator for the KUPOL project and has been responsible for the planning and execution of data collection. All coauthors planned the manuscript together. MA conducted the analysis and drafted the manuscript with continuous feedback and input from MRG, KDL and KK. All coauthors approve the final version of this manuscript.

Funding Open Access funding provided by Karolinska Institute. The Kupol study is funded by a Grant Number (259-2012-48) which includes funds from the Swedish Research Council Formas, The Swedish Research Council for Health, Working Life and Welfare, and The Swedish Research Council-Vetenskapsrådet.

Data Availability Data is available through application at kupolstudien.se. For more information, contact Maria Rosaria Galanti, the PI of the KUPOL study at rosaria.galanti@ki.se. 


\section{Compliance with Ethical Standards}

Conflict of interest The authors declare that they have no conflict of interest.

Ethical Approval The KUPOL study was approved by the Stockholm Ethics Review Board (Reference Numbers: 2012/1904-31/1 and 2016/1280-32).

Consent for Publication All authors have read and approved the final version of this manuscript for publication.

Open Access This article is licensed under a Creative Commons Attribution 4.0 International License, which permits use, sharing, adaptation, distribution and reproduction in any medium or format, as long as you give appropriate credit to the original author(s) and the source, provide a link to the Creative Commons licence, and indicate if changes were made. The images or other third party material in this article are included in the article's Creative Commons licence, unless indicated otherwise in a credit line to the material. If material is not included in the article's Creative Commons licence and your intended use is not permitted by statutory regulation or exceeds the permitted use, you will need to obtain permission directly from the copyright holder. To view a copy of this licence, visit http://creativecommons.org/licenses/by/4.0/.

\section{References}

Almroth, M. C., László, K. D., Kosidou, K., \& Galanti, M. R. (2018). Association between adolescents’ academic aspirations and expectations and mental health: A one-year follow-up study. European Journal of Public Health, 28(3), 504-509.

Almroth, M., László, K. D., Kosidou, K., \& Galanti, M. R. (2019). Academic expectations and mental health in adolescence: A longitudinal study involving parents' and their children's perspectives. Journal of Adolescent Health, 64(6), 783-789.

Appleton, J., Christenson, S., Dongjin, K., \& Reschly, A. (2006). Measuring cognitive and psychological engagement: Validation of the student engagement instrument. Journal of School Psychology, 44, 427-445.

Bear, G. G., Yang, C., Chen, D., He, X., Xie, J.-S., \& Huang, X. (2018). Differences in school climate and student engagement in China and the United States. School Psychology Quarterly, 33(2), $323-335$

Benner, A. D., \& Mistry, R. S. (2007). Congruence of mother and teacher educational expectations and low-income youth's academic competence. Journal of Educational Psychology, 99(1), 140-153.

Berhanu, G. (2010). Even in Sweden? Excluding the included: Some reflections on the consequences of new policies on educational processes and outcomes, and equity in education. International Journal of Special Education, 25(3), 148-159.

Berkowitz, R., Moore, H., Astor, R. A., \& Benbenishty, R. (2016). A research synthesis of the associations between socioeconomic background, inequality, school climate, and academic achievement. Review of Educational Research, 87(2), 425-469.

Betts, J., Reschly, A. L., Appleton, J. J., Christenson, S. L., \& Hueber, E. S. (2002). A study on the factorial invariance of the student engagement instrument (SEI): Results from middle and high school students. School Psychology Quarterly, 25(2), 84-93.

Bronfenbrenner, U. (1979). The ecology of human development: Experiments by nature and design. Cambridge, MA: Harvard University Press.

Bronfenbrenner, U., \& Morris, P. A. (2006). The bioecological model of human development. In Handbook of child psychology: Theoretical models of human development, (Vol. 1). Hoboken, NJ, US: Wiley.

Check and Connect Study. (2015). 5-point scale student engagement instrument (SEI) administration standardization procedures. http://checkandconnect.umn.edu/docs/SEI\%20Administration, \%20Sco ring,\%20and\%20Results\%20Use\%20ICI.pdf.

Chykina, V., Chung, H. J., \& Bodovski, K. (2016). Great expectations? Variation in educational plans of students in post-socialist Eastern Europe. European Education, 48(1), 43-62. 
Cornell, D., Shukla, K., \& Konold, T. R. (2016). Authoritative school climate and student academic engagement, grades, and aspirations in middle and high schools. American Educational Research Association Open, 2(2), 1-18.

Dimitrova, R., Ferrer-Wreder, L., \& Galanti, M. R. (2016). Pedagogical and social climate in school questionnaire: Factorial validity and reliability of the teacher version. Journal of Psychoeducational Assessment, 34(3), 282-288.

Frostick, C., Phillips, G., Renton, A., \& Moore, D. (2016). The educational and employment aspirations of adolescents from areas of high deprivation in London. Journal of Youth and Adolescence, 45(6), 1126-1140.

Galanti, M. R., Hultin, H., Dalman, C., Engström, K., Ferrer-Wreder, L., Forsell, Y., et al. (2016). School environment and mental health in early adolescence: a longitudinal study in Sweden (KUPOL). BMC Psychiatry, 16(1), 243.

Gregory, A., \& Huang, F. (2013). It takes a village: The effects of 10th grade college-going expectations of students, parents, and teachers four years later. American Journal of Community Psychology, 52(1-2), 41-55.

Grosin, L. (2004). Skolklimat, Prestation och Anpassning i 21 Mellan- och 20 Högstadieskolor. Pedagogiska institutionen: Stockholm University.

Haynes, N. M., Emmons, C., \& Ben-Avie, M. (1997). School climate as a factor in student adjustment and achievement. Journal of Educational and Psychological Consultation, 8(3), 321-329.

Hultin, H., Ferrer-Wreder, L., Dimitrova, R., Karlberg, M., \& Galanti, M. R. (2016). Psychometric properties of an instrument to measure social and pedagogical school climate among teachers PESOC. Scandinavian Journal of Educational Research, 62(2), 287-306.

Hultin, H., Eichas, K., Ferrer-Wreder, L., Dimitrova, R., Karlberg, M., \& Galanti, M. R. (2018). Pedagogical and social school climate: Psychometric evaluation and validation of the student edition of PESOC. Scandinavian Journal of Educational Research, 63(4), 1-17.

Irvin, M. J., Byun, S.-Y., Meece, J. L., Reed, K. S., \& Farmer, T. W. (2016). School characteristics and experiences of African American, Hispanic/Latino, and Native American youth in rural communities: Relation to educational aspirations. Peabody Journal of Education, 91(2), 176-202.

Israelashvili, M. (1997). School adjustment, school membership and adolescents' future expectations. Journal of Adolescence, 20(5), 525-535.

Jia, Y., Konold, T. R., \& Cornell, D. (2016). Authoritative school climate and high school dropout rates. School Psycholgy Quarterly, 31(2), 289-303.

Khattab, N. (2015). Students' aspirations, expectations and school achievement: What really matters? British Educational Research Journal, 41(5), 731-748.

Kirk, C. M., Lewis, R. K., Brown, K., Nilsen, C., \& Colvin, D. Q. (2012). The gender gap in educational expectations among youth in the foster care system. Children and Youth Services Review, 34(9), 1683-1688.

Konold, T. R., \& Cornell, D. (2015). Measurement and structural relations of an authoritative school climate model: A multi-level latent variable investigation. Journal of School Psychology, 53(6), 447-461.

Lindstrom-Johnson, S., Pas, E., \& Bradshaw, C. P. (2016). Understanding the association between school climate and future orientation. Journal of Youth and Adolescence, 45(8), 1575-1586.

Madarasova-Geckova, A., Tavel, P., van Dijk, J. P., Abel, T., \& Reijneveld, S. A. (2010). Factors associated with educational aspirations among adolescents: Cues to counteract socioeconomic differences? BMC Public Health, 10, 154.

Marjoribanks, K. (2002). Family contexts, individual characteristics, proximal settings, and adolescents' aspirations. Psychological Reports, 91(3), 769-779.

Melkman, E., Refaeli, T., \& Benbenishty, R. (2016). An empirical test of a model of academic expectations among youth in residential care. Children and Youth Services Review, 67, 133-141.

Mello, Z. R. (2008). Gender variation in developmental trajectories of educational and occupational expectations and attainment from adolescence to adulthood. Developmental Psychology, 44(4), 1069-1080.

Nitardy, C. M., Duke, N. N., Pettingell, S. L., \& Borowsky, I. W. (2015). Racial and ethnic disparities in educational achievement and aspirations: Findings from a statewide survey from 1998 to 2010. Maternal and Child Health Journal, 19(1), 58-66.

OECD. (2015). Improving schools in Sweden: An OECD perspective. Organisation for Economic Cooperation and Development. Retrieved from http://www.oecd.org/edu/school/Improving-Schools-inSweden.pdf.

Roeser, R. W. (2005). Stage-environment fit theory. In C. B. Fisher and R. M. Lerner (Eds.), Encyclopedia of Applied Developmental Science. Thousand Oaks, California: SAGE Publications, Inc. 
Retrieved from http://sk.sagepub.com/reference/applieddevscience. https://doi.org/10.4135/97814 12950565

Rubie-Davies, C. M. (2006). Teacher expectations and student self-perceptions: Exploring relationships. Psychology in the Schools, 43(5), 537-552.

Smith, M. L., Mann, M. J., Georgieva, Z., Curtis, R., \& Schimmel, C. J. (2016). What counts when it comes to school enjoyment and aspiration in the middle grades. Research on Middle Level Education Online, 39(8), 1-13.

Thapa, A., Cohen, J., Guffey, S., \& Higgins-D'Alessandro, A. (2013). A review of school climate research. Review of Educational Research, 83(3), 357-385.

Wang, M.-T., \& Holcombe, R. (2010). Adolescents' perceptions of school environment, engagement, and academic achievement in middle school. American Educational Research Journal, 47(3), 633-662.

Wiborg, S. (2015). Privatizing education: Free school policy in Sweden and England. Comparative Education Review, 59(3), 473-497.

Wikström, C. (2006). Education and assessment in Sweden. Assessment in Education: Principles, Policy and Practice, 13(1), 113-128.

Yang, C., Sharkey, J. D., Reed, L. A., Chen, C., \& Dowdy, E. (2018). Bullying victimization and student engagement in elementary, middle, and high schools: Moderating role of school climate. School Psychology Quarterly, 33(1), 54-64.

Publisher's Note Springer Nature remains neutral with regard to jurisdictional claims in published maps and institutional affiliations. 